\title{
Swimming immobility and rat REM deprivation: A pilot study on time-delay effects
}

\author{
JAMES HAWKINS, NATHAN H. PHILLIPS, ROBERT F. WELLS, \\ JEAN A. HODGSON, and ROBERT A. HICKS \\ San Jose State University, San Jose, California 95192
}

\begin{abstract}
This paper helps to solve a practical problem in the ongoing sequential validation of an animal model concerned with emotionality and adaptation. The performance measure used involves the swimming activity level of rats on a series of trials. Earlier work with a different (open-field) measure had indicated that heightened activity might occur simply as a result of the REM-deprivation method that we use. A problem in evaluating that work, in the context of our own, is that it involves a very long time-delay interval. Our model is sufficiently complex to make that interval impractical for our use. Here we report a small sample evaluation that documents the effect seen earlier, but which also makes possible a more convenient time delay for future work.
\end{abstract}

Our research group has previously detailed a repeatedmeasures protocol for rats in which the inferred REM status of the animals is directly reflected in their swimming test scores. The report on that procedure provided human clinical referents that were speculatively linked to the laboratory rat outcomes and their analysis (Hawkins, Phillips, Moore, Gilliland, Dunbar, \& Hicks, 1980). At the time, we noted that other requisite experiments would be necessary in order to validate this animal model for more general research use. In subsequent presentations, there has been an independent confirmation of our original data (van Luijtelaar \& Coenen, Note 1). A demonstration that developmental differences in REM status (which were produced by environmental enrichment) are picked up by this test (Hodgson, Fox, Hicks, \& Hawkins, in press) and work showing that the rate of response changes systematically with temperature change in the swimming cylinder (Hawkins, Phillips, Gilliland, \& Hicks, in press).

The key behavioral response upon which the rats are evaluated is known as swimming immobility. It was first described by Porsolt, LePichon, and Jalfre (1977). This is a characteristic posture that rats passively learn to adopt after an initial period of vigorous swimming activity. For control animals (using our procedure), this immobility tends to increase both within the standard 5-min trial and across the 7 days of repeated testing. On the other hand, REM-deprived animals do not show very much of this immobility until after they

This report was supported by Grant NIH/MBS RR08192. We thank John Empey and Dougall McDonald for apparatus construction. Reprints may be obtained from Dr. James Hawkins, Department of Psychology, San Jose State University, San Jose, California 95192. are allowed uninterrupted sleep. After such rest, however, their immobility scores are found to approach those of the control group animals (Hawkins et al., 1980). An interpretive problem arises because of Pearlman's (1971) finding that there is transient hyperactivity on an openfield test immediately after platform REM deprivation. Here, we will report findings for the rat swimming test.

Logistically, our elaboration of the swimming test can be somewhat difficult for the incorporation of lengthy, time-based, independent variables. There are three necessary treatment conditions, and testing must occur on each of 7 consecutive days. The reader will appreciate that when we wished to replicate the 3-h delay of Pearlman (1971) as one of three levels of testing (i.e., $3 \mathrm{~h}, 30 \mathrm{~min}$, no delay), data gathering became something of a heroic undertaking. We, therefore, decided to run one animal from each of the three treatment groups at each of the three time-delay levels. For each of the independent variables, then, we could collect data points based on $n=3$ subjects. Since the original swimming immobility measure had been successfully used with very small sample sizes (i.e., $n=5$ in Porsolt et al., 1977), we decided that if the trend lines replicated our earlier work, we would have sufficient basis for needed generalization. Later, a larger sample experiment in which the time delay might cover a narrower range would then be feasible.

\section{METHOD}

We used male Sprague-Dawley rats (Simonsen, Gilroy, California), 40 days old on the 1 st day of testing. REM deprivation was produced by the platform method, using either a small $(6.5-\mathrm{cm})$ or a large $(16.5-\mathrm{cm})$ platform. The dry control (DC) condition was a dry bucket with a large platform in place. The small-platform (SP) condition was a water-filled bucket that 
Table 1

Immobility Means and Standard Deviations for the REMD Treatment and Time-Delay Variables Across the 7 Days of Testing

Trial Day

\begin{tabular}{|c|c|c|c|c|c|c|c|c|c|c|c|c|c|c|}
\hline & \multicolumn{2}{|c|}{1} & \multicolumn{2}{|c|}{2} & \multicolumn{2}{|c|}{3} & \multicolumn{2}{|c|}{4} & \multicolumn{2}{|c|}{5} & \multicolumn{2}{|l|}{6} & \multicolumn{2}{|c|}{7} \\
\hline & Mean & SD & Mean & SD & Mean & SD & Mean & SD & Mean & SD & Mean & SD & Mean & SD \\
\hline & \multicolumn{14}{|c|}{ Treatment } \\
\hline DC & 35.47 & 31.29 & 81.83 & 108.45 & 113.33 & 116.53 & 129.50 & 76.18 & 116.50 & 8.66 & 191.00 & 90.44 & 177.00 & 77.63 \\
\hline LP & 51.47 & 56.17 & 81.80 & 112.04 & 178.50 & 125.67 & 154.67 & 76.83 & 178.33 & 103.49 & 226.00 & 95.19 & 234.00 & 47.31 \\
\hline \multirow[t]{2}{*}{ SP } & 93.33 & 115.93 & 125.67 & 38.35 & 93.33 & 42.00 & 53.83 & 1.04 & 72.17 & 56.64 & 145.83 & 19.75 & 228.67 & 21.51 \\
\hline & \multicolumn{14}{|c|}{ Time Delay } \\
\hline $3 \mathrm{~h}$ & 18.93 & 11.57 & 49.97 & 62.86 & 136.67 & 111.30 & 111.67 & 98.60 & 165.50 & 58.28 & 214.67 & 54.02 & 227.67 & 26.74 \\
\hline $30 \mathrm{~min}$ & 70.13 & 44.84 & 169.00 & 69.31 & 187.00 & 110.12 & 143.00 & 79.18 & 130.67 & 104.58 & 236.00 & 78.35 & 247.00 & 19.49 \\
\hline No Delay & 91.20 & 118.17 & 70.33 & 82.42 & 61.50 & 24.58 & 83.33 & 36.13 & 70.83 & 36.23 & 112.17 & 16.43 & 165.00 & 71.36 \\
\hline
\end{tabular}

Note-For each value, $n=3$.

contained a small plaform. The large-platform (LP) condition was a water-filled (control) bucket that contained a large platform. Further detail on the apparatus has been reported (Hicks \& Moore, 1979). SP existence leads to reduction in REM sleep time because, upon entering REM sleep, the animal.loses muscle tone and falls toward or into the water. It should be noted, however, that most rats (and all that are used experimentally) learn to respond to postural cues and (usually) avoid actual loss of position. LP conditions allow the animal to better situate itself, so that the postural muscle atonia of REM sleep is much less likely to produce even occasional immersion in the water. Theoretically, REM sleep periods should then continue. Still, it is known that LP animals may also lose some REM sleep, especially in the early days of treatment (Mendelson, Guthrie, Frederick, \& Wyatt, 1974). In SP conditions, we infer a $50 \%$ reduction in daily REM sleep at the end of 4 days. Rats in DC do not lose REM sleep (Mendelson et al., 1974). As part of the factorial design, the animals were also tested after a 0 -min, 30-min, or 3-h delay following removal from the buckets. During this interval, they were kept in a total sleep-deprivation device (Mendelson, Stefurak, Stefurak, Gillin, \& Wyatt, 1977) that maintained their relative degree of REM sleep deprivation. This device has been electrophysiologically validated.

The swimming cylinder consisted of a clear Plexiglas tube (height $40 \mathrm{~cm}$, diameter $18 \mathrm{~cm}$ ). It sat in a water bath that maintained both the $25^{\circ} \mathrm{C}$ temperature and the $15-\mathrm{cm}$ water depth at which the rats swim in the standardized test. In this set-up, the rats are able to touch bottom but are also able to swim very briskly, and they may even dive (Hawkins, Hicks, Phillips, \& Moore, 1978). Quickly, however, they begin to learn the energy-saving immobility response. Swimming immobility was defined as the number of seconds that both front feet were extended and in contact with the cylinder wall, with the animal moving neither left nor right. Baseline values were recorded as Day 1. The treatment period for REM deprivation was 4 days and ended with Day 5. All rats then returned to the recovery sleep conditions of DC while tests continued for Days 6 and 7.

\section{RESULTS AND DISCUSSION}

The results are shown in Table 1. In the upper part of the table, the data are arranged to show the effects of the three REM-deprivation treatment conditions. Outcomes from earlier work are confirmed, in that both DC and LP control rats show increasing time in immobility across trials. The REM sleep-deprived SP rats do not do so until they have had consolidated sleep. On Days 6 and 7 (now with uninterrupted DC sleep), the SP scores improve markedly to values well within the control range. A difference in these data from an earlier outcome is that, here, the LP control starts out slightly higher than DC and maintains its rank across trials. In Hawkins et al. (1980), the LP group showed a more intermediate response.

The lower part of Table 1 shows the same data recast to reflect the time-delay variable. A clear-cut effect is seen. Only the no-delay group shows a drop in immobility on Day 2. Further, on Days 3-7, it is both lower and has a different trend line from the other two groups. When the baseline differences between the 3-h and 30-min groups are taken into account, they are seen to be essentially alike in their response. With the recovery sleep of Days 6 and 7, the no-delay group shows higher immobility but still lags well behind both groups of time-delayed rats.

We suspect that time delay might be similar to temperature variation in setting a level of response while leaving REM-deprivation treatment differences intact (Hawkins et al., in press). Still to be determined is whether the swimming test measures might be more informative at some particular time-delay interval rather than in immediate testing. From the present data, we conclude that such an independent variable can have levels of $30 \mathrm{~min}$ and less. This outcome should make more adequate data gathering feasible.

\section{REFERENCE NOTE}

1. van Luijtelaar, E. L. J. M., \& Coenen, A. M. L. A possible antidepressant effect of paradoxical sleep deprivation in rats. Paper presented at the annual meeting of the Association for the Psychophysiological Study of Sleep, June 17-21, 1981, Hyannis, Massachusetts.

\section{REFERENCES}

Hawkins, J., Hicks, R. A., Phillips, N., \& Moore, J. D. Swimming rats and human depression. Nature, 1978, $274,512$. Hawkins, J., Phillips, N. H., Gilliland, M. A., \& Hicks, R. A. Rat swimming and REMD: Water temperature variation. Sleep Research, in press.

Hawkins, J., Phillips, N., Moore, J. D., Gilliland, M. A., Dunbar, S., \& Hicks, R. A. Emotionality and REMD: A rat swimming model. Physiology \& Behavior, 1980, 25, 167-171. 
Hicks, R. A., \& Moore, J. D. REM sleep deprivation increases aggressiveness in male rats. Physiology \& Behavior, 1979, 22, $1097-1100$.

Hodgson, J. A., Fox, R., Hicks, R. A., \& Hawkins, J. REM sleep of enriched and impoverished rats: Adaptation during the swimming test. Sleep Research, in press.

Mendelson, W. B., Guthrie, R. D., Frederick, G., \& WyaTT, R. J. The flower pot technique of rapid eye movement (REM) sleep deprivation. Pharmacology, Biochemistry and Behavior, 1974, 2, 553-556.
Mendelson, W. B., Stefurak, S. J., Stefurak, M. L., Gillin, J. C., \& WyATT, R. J. EEG documentation of a method for sleep depriving rats. Sleep Research, 1977, 6, 203.

Pearlman, C. A. Latent learning impaired by REM sleep deprivation. Psychonomic Science, 1971, 25, 135-136.

Porsolt, R. D., LePichon, M., \& Jalfre, M. Depression: A new animal model sensitive to antidepressant treatments. Nature, 1977, 266, 730-732.

(Received for publication July 24, 1981.) 